$01,07,13$

\title{
Базовые элементы структуры границ зерен наклона. Часть 2. Оси разориентации [110] и [111]*
}

\author{
(C) А.В. Векман, Б.Ф. Демьянов \\ Алтайский государственный технический университет им. И.И. Ползунова, \\ Барнаул, Россия \\ E-mail: weckman@list.ru
}

Поступила в Редакцию 26 февраля 2020 г.

В окончательной редакции 12 марта 2020 г.

Принята к публикации 2 сентября 2020 г.

\begin{abstract}
Методами компьютерного моделирования проведен расчет структуры и энергии симметричных границ зерен (ГЗ) наклона с осями разориентации [110] и [111]. Расчеты выполнялись с использованием структурновакансионной модели. Исследование атомной структуры проведено во всем диапазоне углов разориентации. Специальные границы зерен имели обратную плотность совпадающих узлов $\Sigma \leq 57$. Расчет проводился с использованием парного потенциала Морзе и многочастичного потенциала Клери-Розато. Зависимости энергии ГЗ от угла разориентации, полученные при расчетах разными потенциалами, имеют похожий вид, а атомная структура полностью совпадает. Показано, что структура любой ГЗ с осями разориентации [110] и [111] может быть представлена ограниченным числом базовых структурных элементов. Все обнаруженные базовые структурные элементы, обозначенные элементами типа $A, B, C$ и $D$, основаны на структурах специальных границ. Для ГЗ с осью разориентации [110] такими специальными ГЗ являются $\Sigma 3(111)$, $\Sigma 3(112), \Sigma 11(113)$ и $\Sigma 9(114)$, для Г3 с осью разориентации [111] $-\Sigma 3(112), \Sigma 7(123)$ и $\Sigma 13(134)$. Определены диапазоны углов, в пределах которых встречаются те или иные базовые структурные элементы.
\end{abstract}

Ключевые слова: компьютерное моделирование, граница зерен, структурно-вакансионная модель, базовые структурные элементы.

DOI: 10.21883/FTT.2021.01.50398.398

\section{1. Введение}

Исследование атомной структуры границ зерен (ГЗ) до сих пор остается актуальной проблемой в силу значительного влияния ГЗ на свойства поликристаллов, a также большого разнообразия видов ГЗ: наклона и кручения; специальные и общие; симметричные и асимметричные. По этой причине для исследования ГЗ применяется большое количество моделей. Наиболее часто используемые модели решетки совпадающих узлов (РСУ), структурных единиц (СЕ) и полиэдры Бернала были созданы более 50 лет назад. С тех пор было проведено множество экспериментальных и теоретических исследований структуры ГЗ, которые показали, что реальные атомные конфигурации ГЗ гораздо более сложные, чем описанные в этих моделях. Тем не менее, современные компьютерные расчеты в той или иной мере опираются на эти модели [1].

Исторически модель СЕ была предложена в работе Бишопа и Чалмерса в 1968 [2] на примере ГЗ наклона с осью разориентации [100]. С этого времени атомной структуре границ зерен посвящено немало теоретических и экспериментальных работ. Однако в большинстве из них исследуются границы с осями разориентации [100], причем наибольшее внимание уделяется специальным ГЗ с малыми значениями обратной плотности

\footnotetext{
*) Часть 1. См. ФТТ 62. В. 12. С. 1997.
}

совпадающих узлов $\Sigma$. Исследования ГЗ с осью разориентации [110] и [111] встречаются в литературе гораздо реже.

Впервые анализ структуры границ наклона с осью разориентации [110], используя модель СЕ, был проведен в работе [3]. Моделирование проводилось с использованием парного потенциала. Половинки бикристалла поворачивались относительно общей оси, после чего проводилась релаксация путем смещения, как отдельных атомов, так и половинок относительно друг друга вдоль оси разориентации. Авторы провели исследование в двух угловых диапазонах $0^{\circ} \leq \Theta \leq 31.59^{\circ}$ и $31.59^{\circ} \leq \Theta \leq 50.48^{\circ}$. ГЗ $\Sigma 27(115)$, угол разориентации которой разделяет эти два диапазона, представлена структурными единицами обозначенными символом $A$. Необходимо отметить, что данная структура отличается от границы в модели РСУ тем, что атомы смещены из узлов кристаллической решетки в результате релаксационной процедуры. Структура границы $\Sigma 11(113)$ с углом разориентации $50.48^{\circ}$ мало отличается от структуры РСУ, а период повторяемости назван авторами структурной единицей $B$. Структурная единица типа $C$ является, по сути, участком идеального кристалла. Граница $\Sigma 1$ с углом разориентации $0^{\circ}$ состоит из таких элементов. В диапазоне углов $0^{\circ}-31.59^{\circ}$ рассчитана структура пяти специальных границ, а в $31.59^{\circ}-50.48^{\circ}$ - семи. В первом диапазоне структура всех границ состоит из элементов $A$ и $C$, а во втором $-A$ и $B$. По мере 
увеличения угла разориентации в первом диапазоне структурные единицы $A$ постепенно „вытесняют“ единицы $C$, а во втором происходит постепенная смена единиц $A$ единицами $B$.

В работе [4] проведено моделирование специальных ГЗ во всем диапазоне углов разориентации $\left(0^{\circ} \leq \Theta \leq 180^{\circ}\right)$. Всего рассмотрена структура 23 -х границ. Границы содержали пять структурных единиц $A$, $B, C, D$ и $E$. Элемент $A$, представляет структуpy $\Sigma 1(001), B-\Sigma 27(115), C-\Sigma 11(113), D-$ $\Sigma 3(111)$ и $E-\Sigma 9(221)$. Использование относительного сдвига соседних зерен для релаксации границ привело к тому, что одна и та же граница может быть образована разным набором структурных единиц. Например, бессдвиговая структура ГЗ $\Sigma 27(115)$ представлена только единицами $B$, тогда как использование относительного сдвига зерен привело к структуре $|A B C A B C|$. Авторы выделяют четыре диапазона углов: $0^{\circ} \leq \Theta \leq 50.48^{\circ}, 50.48^{\circ} \leq \Theta \leq 109.47^{\circ}$, $109.47^{\circ} \leq \Theta \leq 141.06^{\circ}$ и $141.06^{\circ} \leq \Theta \leq 180^{\circ}$. В первом границы содержат элементы $A, B, C$ и $D$; во втором $A, C$ и $D$; в третьем $D$ и $E$; в четвертом - $A$ и $E$.

Неоднозначность в интерпретации структуры границ прослеживается и в других работах. Например, при изучении специальной ГЗ $\Sigma 9(221)$ с помощью просвечивающей электронной микроскопии высокого разрешения [5] авторы строят симметричный участок границы с одной стороны как чередование элементов $D$ и $E$, а с другой $-A$ и $E$.

Нами также было проводилось исследование структуры двадцати специальных ГЗ с осью разориентации [110] в рамках модели CE [6]. В работе было обнаружено, что структура границ содержит элементы четырех границ $\Sigma 3(111), \Sigma 3(112), \Sigma 11(113)$ и $\Sigma 9(114)$, а некоторые элементы могут иметь несколько модификаций, которые получаются внесением в область ГЗ атомов или вакансий.

Еще более сложную атомную структуру имеют ГЗ с осью разориентации [111]. Первое исследование ГЗ такого типа в рамках модели структурных единиц было проведено в работе [3]. Было исследовано девять специальных Г3 с параметром $\Sigma 57$ и углами разориентации от $0^{\circ}$ до $60^{\circ}$. Выделено 6 структурных единиц, из которых состоят эти границы. Элементы $A$ представляют собой единичный элемент идеального кристалла, a элемент $B^{*}$ - тетраэдр. Из этих двух элементов авторы составили границы $\Sigma 13, \Sigma 21, \Sigma 31, \Sigma 43$ и $\Sigma 57$ с отрицательными значениями относительного сдвига зерен. Для границ $\Sigma 21$ и $\Sigma 13$ существует также положительный относительный сдвиг зерен, приводящий к устойчивой структуре. В этом случае авторы описывают эти границы элементами $C$ и $D$ соответственно, которые имеют вид тригональных призм. Границы $\Sigma 7$ и $\Sigma 3$ составлены из элементов $E$ и $F$ также имеющих вид тригональных призм, а границы $\Sigma 19$ и $\Sigma 37$, углы которых имеют значения больше чем у $\Sigma 7$ и меньше чем у $\Sigma 3$, также составлены из этих элементов. Граница
$\Sigma 3$ имеет два устойчивых состояния с положительным и отрицательным относительным сдвигом. При этом в первом случае она составлена из элементов $F$, а во втором из элементов $G$, имеющего вид тетраэдра.

В работе [7] авторы исследуют симметричные границы с осью разориентации [111] с углами разориентации от $20^{\circ}$ до $60^{\circ}$. При этом все эти границы составлены из элементов $E, F$ и $F^{\prime}$. Элемент $E$ является участком идеального кристалла, представленным в работе как границей $\Sigma 1$, а $F$ и $F^{\prime}$ двумя устойчивыми структурами границы $\Sigma 1$. Элемент $F^{\prime}$ отличаются от $F$ тем, что в результате релаксационной процедуры в нем появляется дополнительный атом. Таким образом, описание ГЗ в рамках модели структурных единиц зависит от применяемой методики моделирования и приводит к неоднозначным результатам.

В 1989 и 1990 годах Вольф опубликовал серию работ [8-11], в которой наряду с границами с осями разориентации [100] и [110] изучались 11 специальных ГЗ с осью разориентации [111]. Работы проводились методом компьютерного моделирования на основе модели решетки совпадающих узлов (РСУ) и были посвящены изучению зависимости энергии границ от угла разориентации. Автор пришел к выводу, что энергия ГЗ увеличивается линейно с повышением избыточного объема. Подобные результаты получены в работе [12], в которой проведено исследование методом компьютерного моделирования избыточного объема ГЗ в меди и никеле. Была построена зависимость энергии границ от угла разориентации в целом повторяющая зависимости, полученные Вольфом. Авторы исследования [13] изучали сдвиговое движение для большого числа ГЗ, включая 11 симметричных границ наклона с осью разориентации [111]. В работе [14] проведено тщательное исследование высокотемпературного поведения $\Sigma 7$ с использованием метода молекулярной динамики. Были рассчитаны энергии Гиббса в широком диапазоне температур, вплоть до температуры плавления. Авторы наблюдали фазовый переход первого рода на границе зерен в двумерном и трехмерном бикристаллах при температурах значительно ниже температуры объемного плавления. Детальное изучение структуры и энергии шести границ, в том числе двух $(\Sigma 7$ и $\Sigma 13)$ с осью разориентации [111] проведено в работе [15].

В первой части нашего исследования [16] нами было введено понятие базовых структурных элементов ГЗ. На примере ГЗ с осью разориентации [100] было показано, что любую границу можно рассматривать как чередование ограниченного числа элементов структуры, состоящих из небольшого числа атомов - базовых структурных элементов. Объемный вид базовых структурных элементов зависит от типа границы и оси разориентации. Целью данной работы является изучение структуры симметричных ГЗ наклона с осями разориентации [110] и [111] исходя из идеи базовых структурных элементов. 

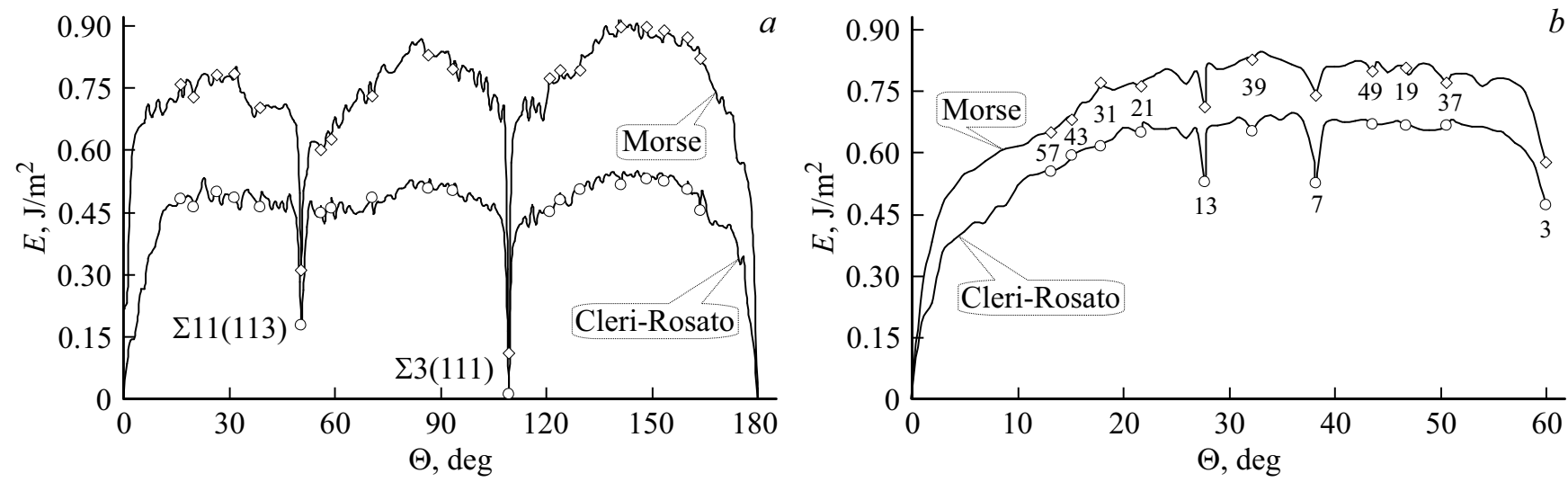

Рис. 1. Зависимости энергии ГЗ от угла разориентации: $a-$ ось разориентации [110], $b-$ ось разориентации [111].

\section{2. Модель}

Исследование проведено с использованием разработанной нами структурно-вакансионной модели ГЗ. Основные положения модели изложены в работах $[1,17,18]$. Данная модель позволяет определять атомную структуру ГЗ наклона общего и специального типов. В соответствии со структурно-вакансионной моделью, исходная конфигурация атомов ГЗ с осью разориентации [110] получается поворотом двух половинок кристалла вокруг общей кристаллогеометрической оси, как это делается в модели РСУ. Особенность построения ГЗ с осью разориентации [111] состоит в том, что кристалл состоит из чередования трех плотноупакованных плоскостей. Поэтому если просто повернуть две половинки идеального кристалла симметричная ГЗ не получается. В связи с этим для создания симметричной границы приходилось зеркально отображать одну половинку кристалла относительно плоскости ГЗ и только после этого проводить поворот. После такого построения часть атомов в области ГЗ находятся на расстоянии, отличающемся от равновесного, в результате чего граница имеет высокое значение энергии. По определенной процедуре в область ГЗ вводятся дополнительные атомы и вакансии, которые стабилизирует структуру границы и значительно понижает ее энергию. Данная процедура называется вакансионной релаксацией $[19,20]$. После вакансионной релаксации проводится смещение атомов под действием межатомных сил, которое дополнительно понижает энергию ГЗ и стабилизирует ее структуру. Расчеты проводились с использованием парного межатомного потенциала Морзе [21] и многочастичного потенциала КлериРозато [22]. Параметры потенциалов соответствовали алюминию и были взяты из [23] для потенциала Морзе и [22] для потенциала Клери-Розато. Сравнение результатов, полученных с применением различных потенциалов, позволяет оценить адекватность структурно-вакансионной модели для нахождения атомной структуры ГЗ.

Угол разориентации $\Theta$ симметричных границ зерен наклона с осью разориентации [110] изменяется в преде- лах от $0^{\circ}$ до $180^{\circ}$, а для оси разориентации [111] в диапазоне от $0^{\circ}$ до $60^{\circ}$. Как было показано в работах $[24,25]$ лишь небольшое количество ГЗ, которые с точки зрения модели РСУ являются специальными, проявляют особые свойства. В частности эти границы имеют низкие значения зернограничной энергии, а обратная плотность узлов совпадения $\Sigma$ имеет небольшие значения. Нами был проведен расчет энергии симметричных ГЗ - при этом шаг по углам разориентации составлял один градус. Отдельно были рассчитаны структура и энергия границ специального типа с параметром $\Sigma \leq 51$ и $\Sigma \leq 57$ для осей разориентации [110] и [111] соответственно. Кристаллогеометрические параметры специальных ГЗ приведены в табл. 1.

Таблица 1. Параметры специальных ГЗ: обратная плотность совпадающих узлов $(\Sigma)$; индексы Миллера плоскости ГЗ $(h k l)$ и угол разориентации $(\square)$

\begin{tabular}{c|r|r||r|r|r}
\hline$\Sigma$ & $h k l$ & $\Theta,{ }^{\circ}$ & $\Sigma$ & $h k l$ & $\Theta,^{\circ}$ \\
\hline \multicolumn{5}{c}{ Ось разориентации $[110]$} \\
\hline 3 & 112 & 70.53 & 3 & 111 & 109.47 \\
9 & 114 & 38.94 & 9 & 221 & 141.06 \\
11 & 113 & 50.48 & 11 & 332 & 129.52 \\
17 & 223 & 86.63 & 17 & 334 & 93.37 \\
19 & 116 & 26.53 & 19 & 331 & 153.47 \\
27 & 115 & 31.59 & 27 & 552 & 148.41 \\
33 & 118 & 20.05 & 33 & 441 & 159.95 \\
33 & 225 & 58.99 & 33 & 554 & 121.01 \\
41 & 338 & 55.88 & 41 & 443 & 124.12 \\
51 & 1110 & 16.10 & 51 & 551 & 163.90 \\
\hline \multicolumn{6}{c}{ Ось разориентации [111] } \\
\hline 3 & 112 & 60.00 & 37 & 347 & 50.57 \\
7 & 123 & 38.21 & 39 & 257 & 32.20 \\
13 & 134 & 27.80 & 43 & 167 & 15.18 \\
19 & 235 & 46.83 & 49 & 358 & 43.57 \\
21 & 145 & 21.79 & 57 & 178 & 13.17 \\
31 & 156 & 17.90 &
\end{tabular}




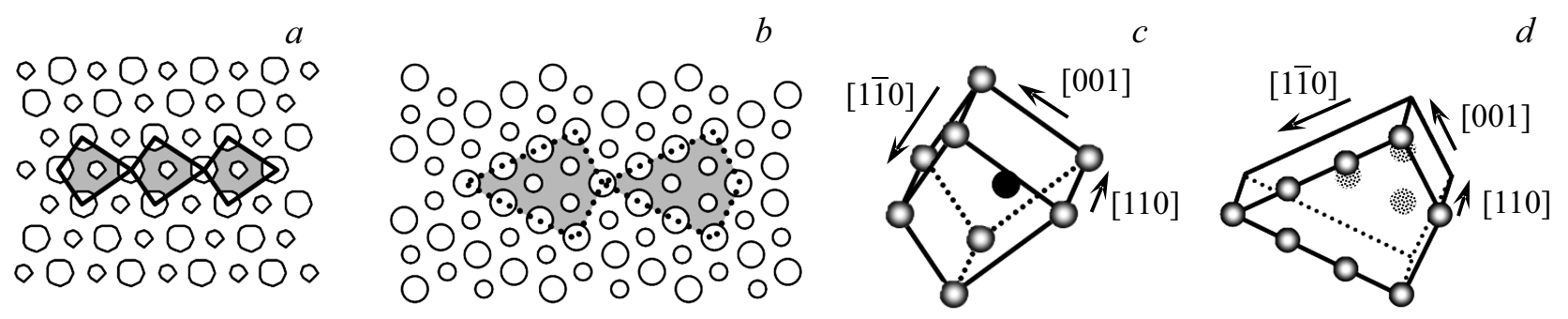

Рис. 2. Структура специальных Гз $\Sigma 3(111)(a)$ и $\Sigma 11(113)(b)$ и базовые структурные элементы типа $A(c)$ и типа $C(d)$.
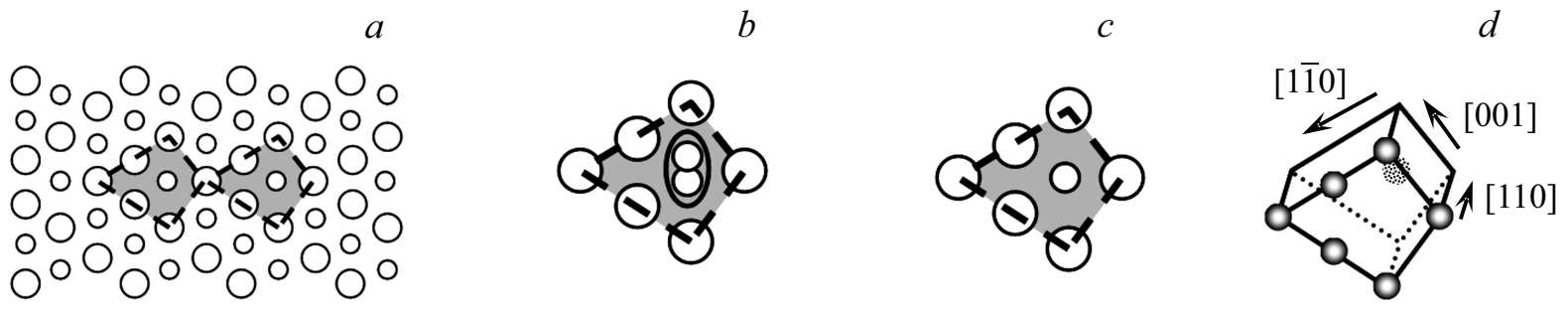

Рис. 3. Структура специальной ГЗ $\Sigma 3(112)(a)$, структурный элемент типа $B$ в модели РСУ $(b)$, его модификация $(c)$ и объемный вид этой модификации $(d)$.

На рис. 1 представлены зависимости энергии стабильных ГЗ полученные с использованием потенциалов Морзе и Клери-Розато. Специальные границы выделены на зависимостях маркерами. Как видно из рисунка зависимости имеют подобный вид, отсутствуют глубокие провалы энергии для специальных ГЗ, за исключением двух границ с осью разориентации [110] $-\Sigma 11(113)$ и $\Sigma 3(111)$, и трех границ с осью разориентации [111] $\Sigma 3(112), \Sigma 7(123)$ и $\Sigma 13(134)$. Кривая, рассчитанная с использованием потенциала Морзе, дает более высокие значения энергии по сравнению с потенциалом Клери-Розато. Величина энергии большеугловых ГЗ, полученная с использованием потенциала Морзе лежит в диапазоне $0.60-0.85 \mathrm{~J} / \mathrm{m}^{2}$ для обеих осей разориентации. Энергии границ полученных с использованием потенциала Клери-Розато отличаются в зависимости от оси разориентации. Для ГЗ с осью разориентации $[110]-0.40-0.55 \mathrm{~J} / \mathrm{m}^{2}$, а для границ с осью разориентации $[111]-0.50-0.65 \mathrm{~J} / \mathrm{m}^{2}$. Экспериментальное значение энергии ГЗ для алюминия составляет $0.60 \mathrm{~J} / \mathrm{m}^{2}$ [26], т.е. энергия, полученная с применением парного потенциала является завышенной, а многочастичного - заниженной. Энергетические зависимости, изображенные на рис. 1 хорошо согласуются с исследованиями других авторов $[4,11,13,27]$.

Сравнение координат атомов, рассчитанных с использованием потенциалов Морзе и Клери-Розато, показало их совпадение с высокой точностью. Различие не превышает $3 \%$ от радиуса первой координационной сферы, что свидетельствует о слабом влиянии вида потенциала на структуру ГЗ. Такой же результат был получен при расчетах ГЗ с осью разориентации [100] в работе [28].

\section{3. Структура границ зерен с осью разориентации [110]}

Поиск базовых структурных элементов начнем с ГЗ с самыми низкими зернограничными энергиями, т.е. $\Sigma 3(111)$ и $\Sigma 11(113)$. На рис. 2, $a$ и $b$ изображены участки этих границ после всех видов релаксационных процедур. Серым цветом ограничены элементы границ в модели РСУ, а линиями разного типа группы атомов, являющиеся базовыми структурными элементами типа $A$ и $C$. Объемный вид этих элементов представлен на рис. $2, c$ и $d$. Видно, что границы $\Sigma 3(111)$ и $\Sigma 11(113)$ стабильны в модели РСУ. По этой же причине данные ГЗ имеют низкие значения энергии.

Еще одна граница с малым значением $\Sigma$ - это граница $\Sigma 3(112)$. На рис. 3, $a$ представлена стабильная структура этой ГЗ. Однако данная граница в модели РСУ имеет высокие значения энергии. Ее структурный элемент в модели РСУ, названный базовым элементом $B$, содержит два атома сильно сближенных друг с другом (рис. $3, b)$. На рисунке эти атомы обведены овалом. Удаление одного атома значительно понижает энергию границы и стабилизирует ее структуру. Это эквивалентно введению в структуру одной распределенной вакансии на структурный элемент. Полученная структура и ее объемный вид изображены на рис. $3, c$ и $d$ соответственно.

Границы $\Sigma 41(338)$ и $\Sigma 33(225)$ имеют углы разориентации меньше, чем у $\Sigma 3(112)$ и больше, чем $\Sigma 11(113)$. С точки зрения модели структурных единиц они либо должны иметь собственные атомные структуры, либо состоять из структурных элементов типа $B$ и $C$. Периоды повторяемости этих границ изображены на рис. $4, a$ 

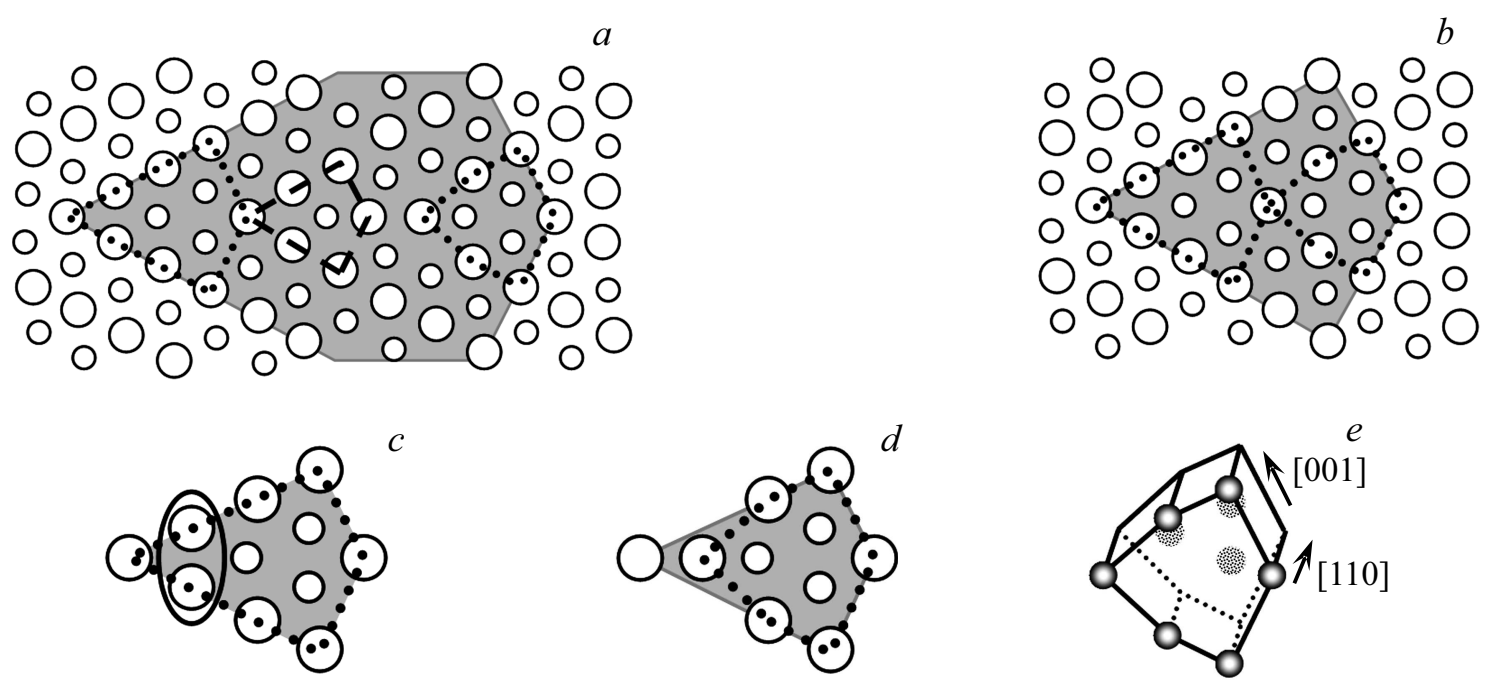

Рис. 4. Структура специальных ГЗ $\Sigma 41(338)(a), \Sigma 33(225)(b)$, структурный элемент типа $C$ в модели РСУ (c), его модификация $(d)$ и объемный вид этой модификации $(e)$.
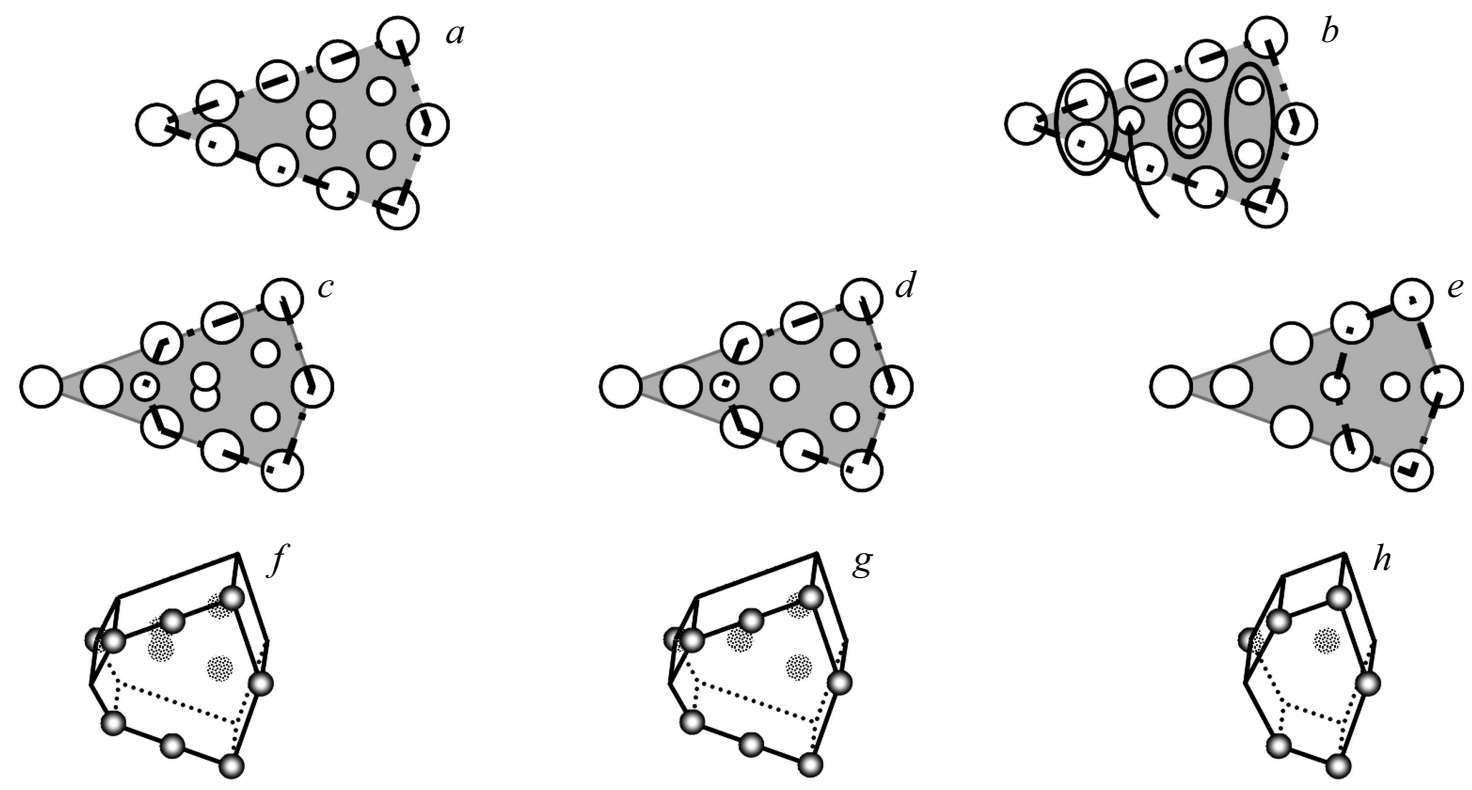

Рис. 5. Структурный элемент типа $D$ в модели РСУ $(a)$, модификации элемента типа $D(b, c, d, e)$ и объемный вид модификаций $(f, g, h)$.

и $b$ соответственно. В данных атомных структурах были найдены базовые элементы $B, C$ и модифицированный элемент, который получается введением одной вакансии в структуру $C$. На рис. 4, $c$ овалом обведены те атомы, которые „объединяются“" в один, а на рис. 4, $d$ и $e$ модификация базового элемент $C$ и ее объемный вид.

Граница $\Sigma 9(114)$ имеет угол разориентации меньше чем у ГЗ $\Sigma 11(113)$. Период повторяемости данной границы в модели РСУ изображен на рис. 5, a. Однако в таком виде граница имеет высокие значения энергии, т.к. в ней существует область, в которой имеется большой избыточный объем. Для стабилизации структуры в эту область необходимо внести дополнительный атом.
На рис. 5, $b$ этот атом отмечен стрелкой. Кроме того, часть атомов в этой структуре является сближенной на расстояние меньше равновесного. На рис. 5, $b$ эти пары атомов обведены овалами. На рис. 5, $c-e$ изображены модификации базового структурного элемента типа $D$ с различным количеством вакансий.

Рассмотрим структуры границ, у которых угол разориентации меньше чем у $\Sigma 9(114)$. Структуры этих границ изображены на рис. 6 в порядке увеличения их углов. Видно, что все они состоят из модификаций структурного элемента типа $D$. Часть структурных элементов смещено на одну плоскость от наблюдателя, что отмечено на рисунках стрелками. Нужно отметить, 

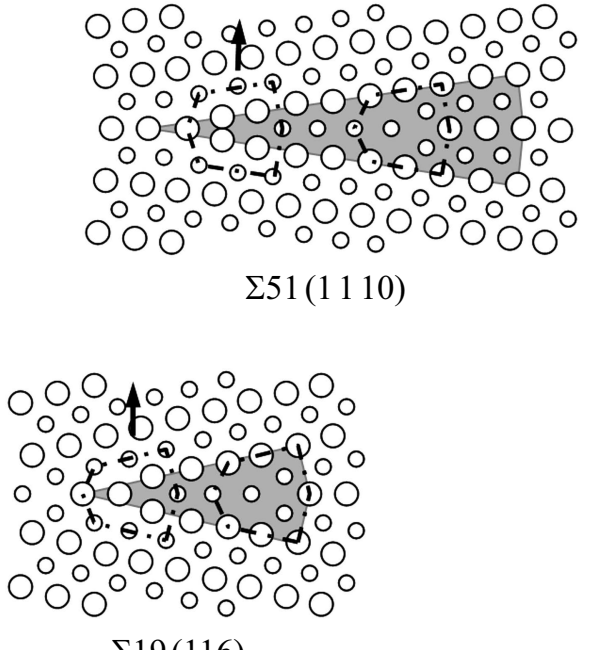

$\Sigma 19(116)$
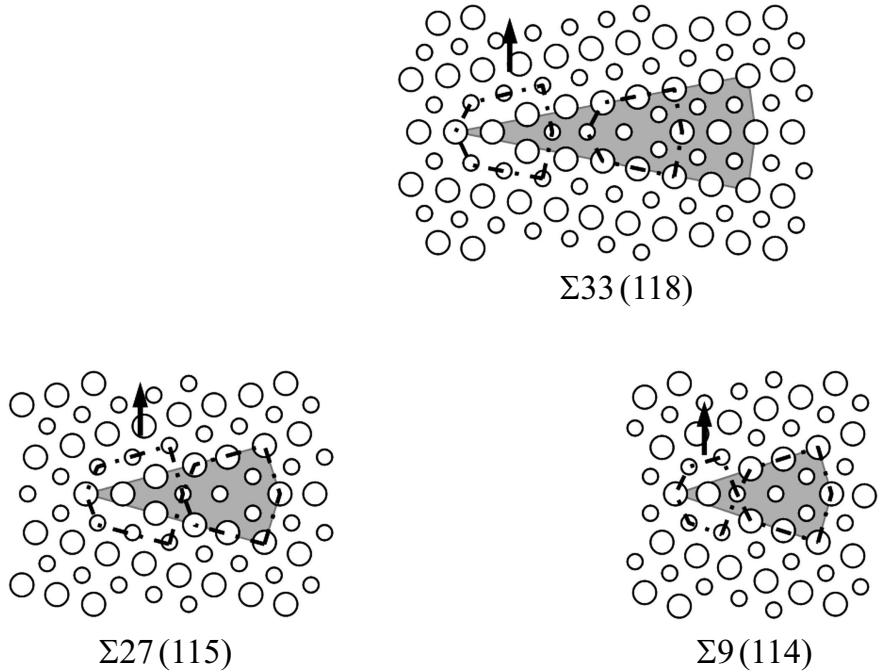

Рис. 6. Структура специальных ГЗ.

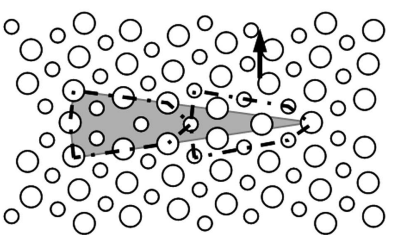

$\Sigma 51(551)$

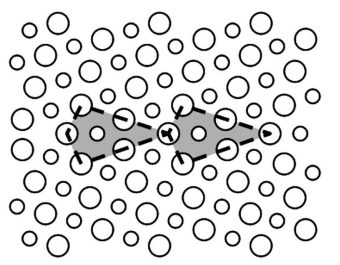

$\Sigma 9(221)$

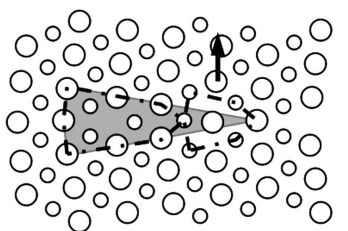

$\Sigma 33(441)$

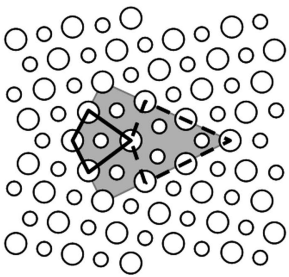

$\Sigma 11(332)$

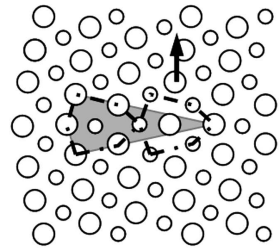

$\Sigma 19(331)$

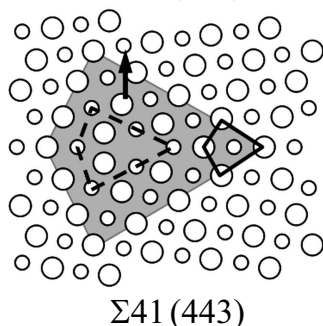

$\Sigma 41(443)$

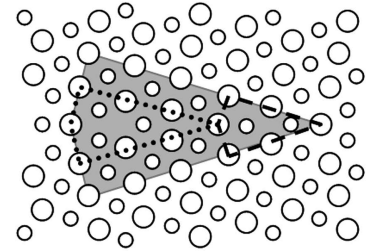

$$
\Sigma 27(552)
$$

00000000000 0000000000000 0000000000000

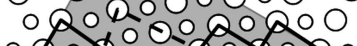

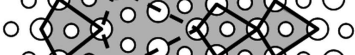
- 10000000 000000000000 000000000000 00000000000 $\Sigma 33(554)$

Рис. 7. Структуры „зеркальных“ ГЗ.

что элементы типа $D$ в чистом виде не встречались, даже граница $\Sigma 9(114)$ может быть представлена только чередованием модификаций этого элемента (рис. 6).

Для всех рассмотренных ГЗ существуют „зеркальные“сраницы с углами разориентации $\Theta>90^{\circ}$. Как видно из табл. 1 эти границы также имеют обратную плотность совпадающих узлов $\Sigma \leq 51$, а если сложить два угла ГЗ с одинаковым $\Sigma$, то получится $180^{\circ}$. Атомные структуры зеркальных ГЗ приведены на рис. 7 в прядке уменьшения угла разориентации. Для этих границ, характерны „зеркальные“ базовые структурные элементы, из которых они состоят. Атомные структуры $\Sigma 51(551), \Sigma 33(441)$ и $\Sigma 19(331)$ содержат ,зеркальные“ модификации элемента $D$.

По мере приближения угла разориентации к 90 в структуре появляется сначала элемент $C$, а потом элементы $B$ и $A$, причем в границе $\Sigma 11(332)$ присутствует модификация элемента $B$ с лишним атомом. Из всего списка, представленного в табл. 1 осталось еще две ГЗ $-\Sigma 17(223)$ и $\Sigma 17(334)$ с углами разориентации $\Theta=86.63^{\circ}$ и $\Theta=93.37^{\circ}$ соответственно. Эти две ГЗ расположены между границами $\Sigma 3(112)$ и $\Sigma 3(111)$. Граница $\Sigma 17(223)$ состоит из элементов $A$ и $B$, а $\Sigma 17(334)$ из элементов типа $A$ и участка почти идеального кристалла.

Поскольку границы специального типа состоят из рассмотренных выше базовых структурных элементов, то логично предположить, что и границы общего типа так же составлены из них. Анализ структуры ГЗ общего типа позволил определить границы углов разориентации, в которых встречаются базовые структурные элементы и их модификации (рис. 8). Из рисунка видно, что любая граница может состоять из одного или двух базовых структурных элементов (включая их модификации). Все малоугловые границы состоят из модификаций базового структурного элемента типа $D$. „Шкала“ базовых структурных элементов симметрична относительно специальной границы $\sigma 3(111)$, а элемент 


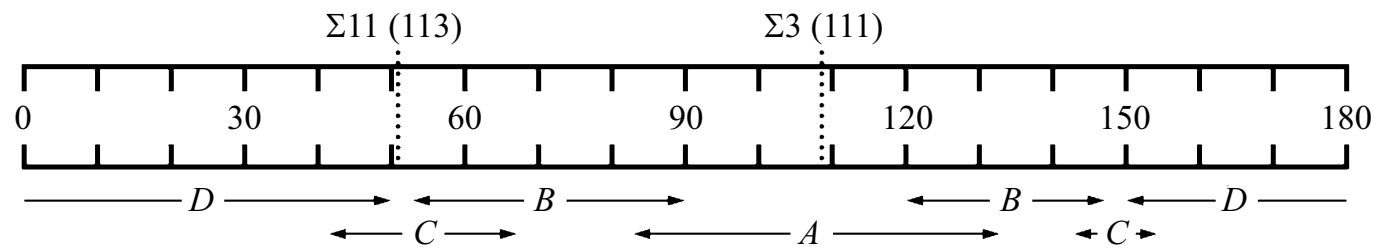

Рис. 8. „Шкала“ базовых структурных элементов ГЗ с осью разориентации [110].
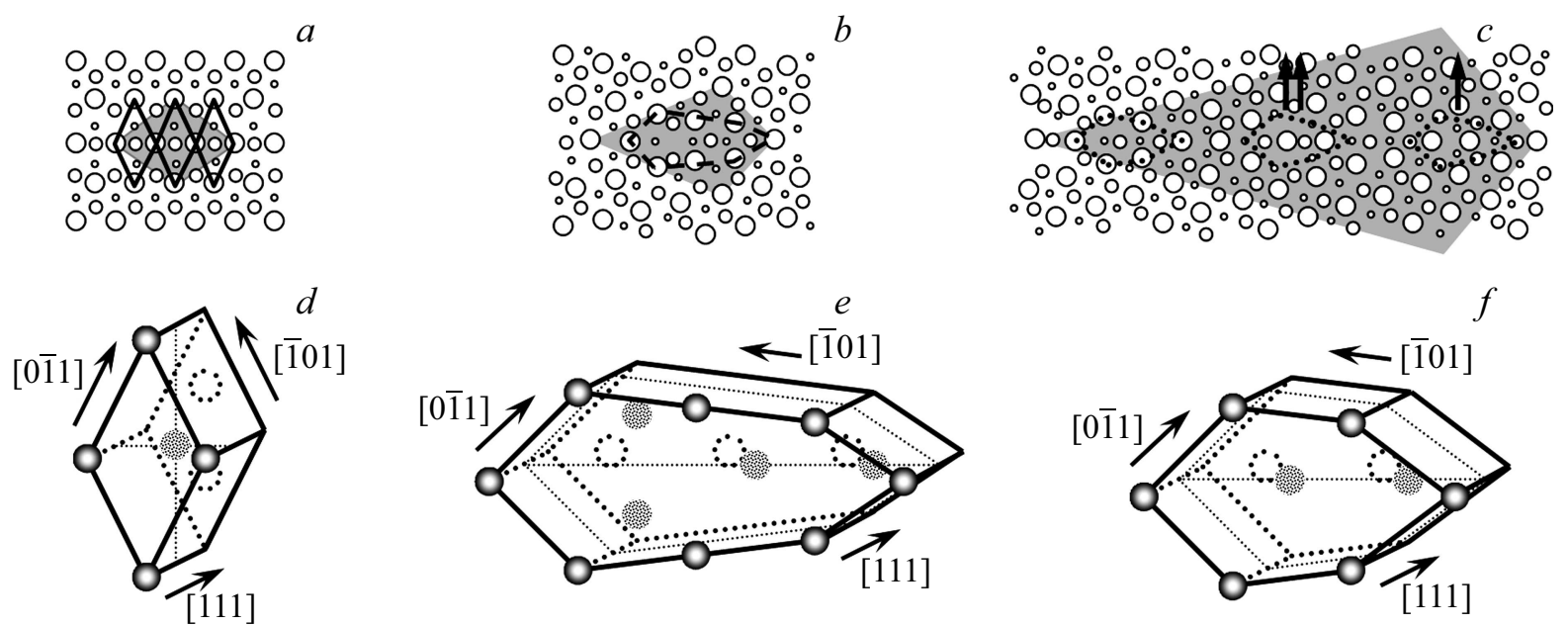

Рис. 9. Структура специальных ГЗ $\Sigma 3(112)(a), \Sigma 7(123)(b)$ и $\Sigma 13(134)(c)$ базовые структурные элементы типа $A$ (d), $B$ (е) и $C$ (f).

типа $C$ находится в самом узком диапазоне углов разориентации, т.е. является промежуточным между элементами типа $B$ и $D$.

Таким образом, проведенный анализ атомной структуры ГЗ наклона с осью разориентации [110] позволил определить базовые структурные элементы этих ГЗ. Обнаружены четыре структуры, изображенные в левом столбце табл. 2 и их модификации. Модификации структурных элементов получаются внесением в структуру дополнительных атомов или вакансий. Серым цветом выделены те базовые элементы, которые в структуре рассмотренных границ не встречались.

\section{4. Структура границ зерен с осью разориентации [111]}

Поиск базовых структурных элементов, как и в случае границ с осью разориентации [110], начнем с ГЗ с самыми низкими зернограничными энергиями, т.е. $\Sigma 3(112)$,

Таблица 2. Базовые структуры границ зерен наклона типа [110]

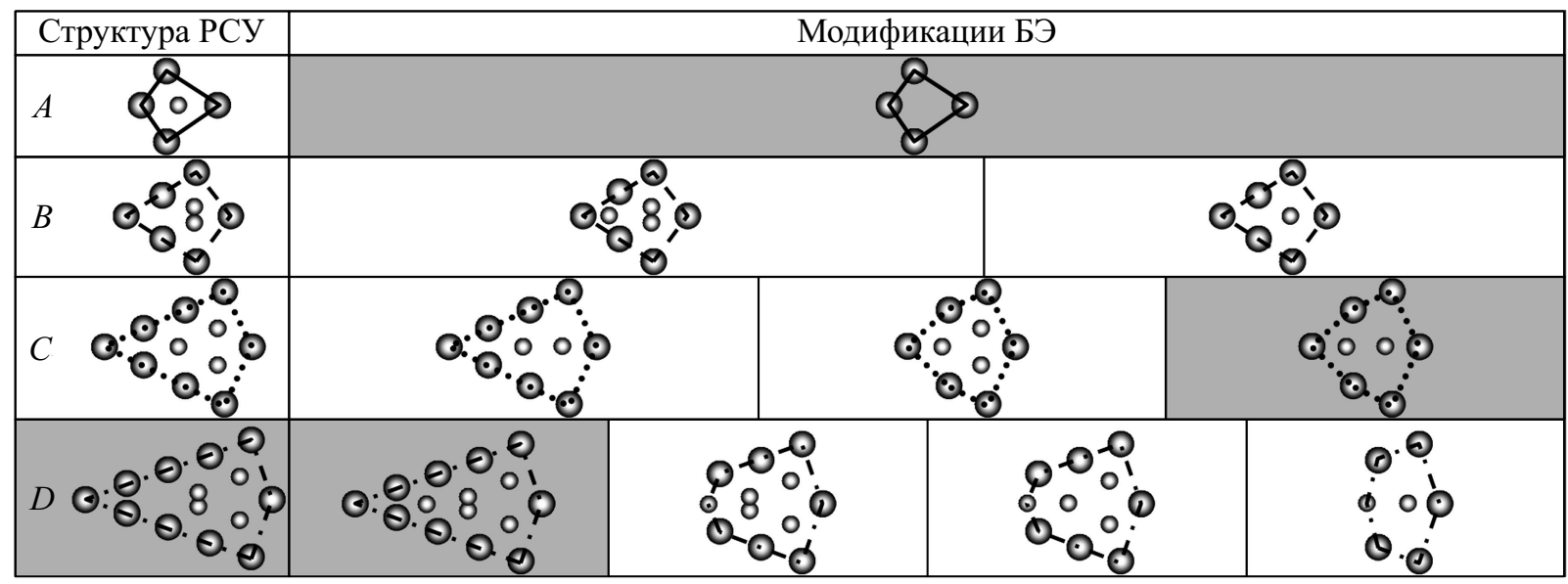




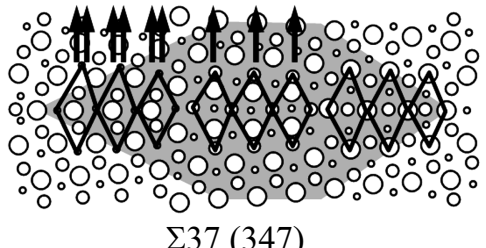

$\Sigma 37$ (347)

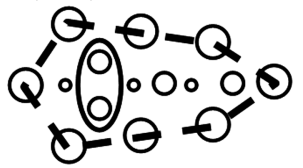

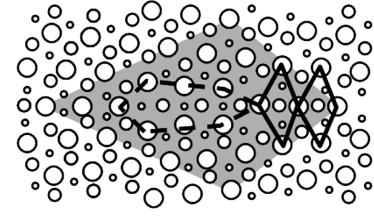

$\Sigma 19(235)$
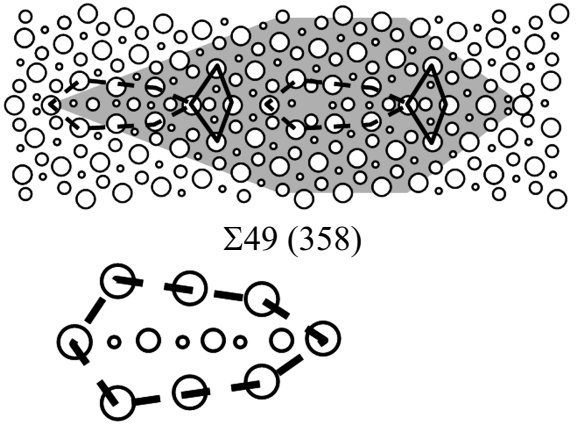

Pис. 10. Структура специальных ГЗ и модификации структурного элемента типа $B$.

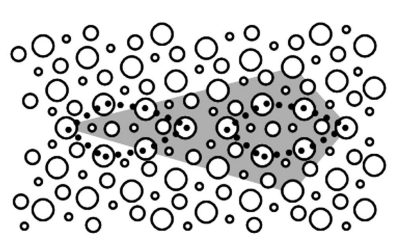

$\Sigma 39$ (257)

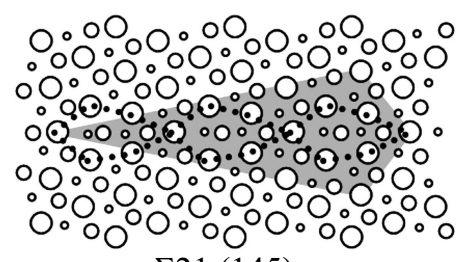

$\Sigma 21(145)$

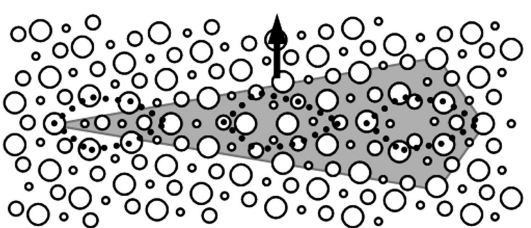

$\Sigma 31$ (156)

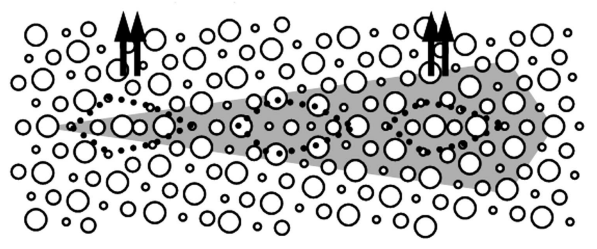

$\Sigma 43(167)$

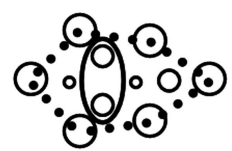

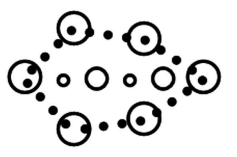

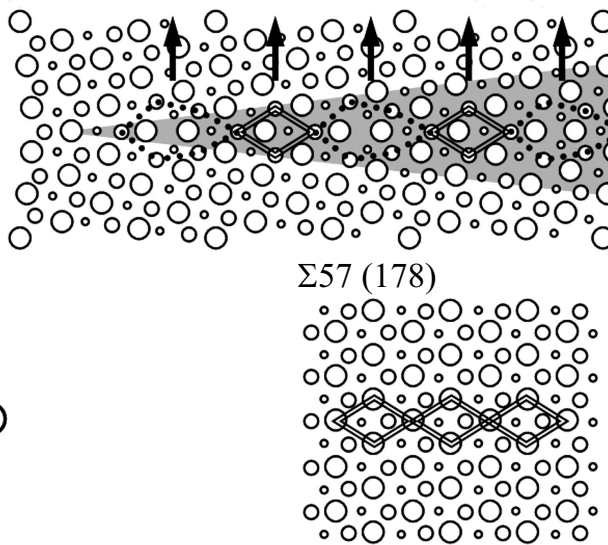

Рис. 11. Структура специальных ГЗ, модификации структурного элемента типа $C$ и участок идеального кристалла.

$\Sigma 37(123)$ и $\Sigma 313(134)$. На рис. 9, $a-c$ изображены участки этих границ после всех видов релаксационных процедур. Серым цветом ограничены элементы границ в модели РСУ, а линиями разного типа группы атомов, являющиеся базовыми структурными элементами типа $A, B$ и $C$. Объемный вид этих элементов представлен на рис. 9, $d-f$. Согласно модели структурных единиц данная конфигурация атомов должны входить и в другие границы. По этой же причине данные ГЗ имеют низкие значения энергии. Стрелка на рис. 9, с означает, что элемент сдвинут на одну плоскость от наблюдателя, а двойная стрелка - что элемент сдвинут на две плоскости. Между структурными элементами прослеживается структура близкая к идеальному кристаллу.

На рис. 10 представлены стабильные структуры ГЗ угол разориентации которых лежит в интервале между углами разориентации границ $\Sigma 7$ и $\Sigma 3$. Границы расположены в порядке уменьшения угла разориентации $\Theta$. По мере уменьшения $\Theta$ в структуре специальных гра- ниц структурные базовые элементы типа $A$ сменяются элементами типа $B$, причем этот элемент имеет две модификации (рис. 10). Одна модификация отличается от другой тем, что два атома объединенные на рис. 10 овалом заменяются одним, расположенным в плоскости границы.

На рис. 11 изображены все остальные специальные ГЗ в порядке уменьшения угла разориентации. Все они состоят из базовых структурных элементов типа $C$. Этот элемент, так же как и элемент $B$, представлен в двух модификациях изображенных на рис. 11. Граница $\Sigma 57(178)$ имеет самый маленький угол разориентации $\Theta=13.17^{\circ}$, т. е. ее можно считать к малоугловой. В структуре этой границы наряду с элементом типа С присутствуют группы атомов, представляющих собой участки идеального кристалла (рис. 11). Данные группы мы назвали базовым структурным элементом типа $D$.

Поскольку границы специального типа состоят из рассмотренных выше базовых структурных элементов, 


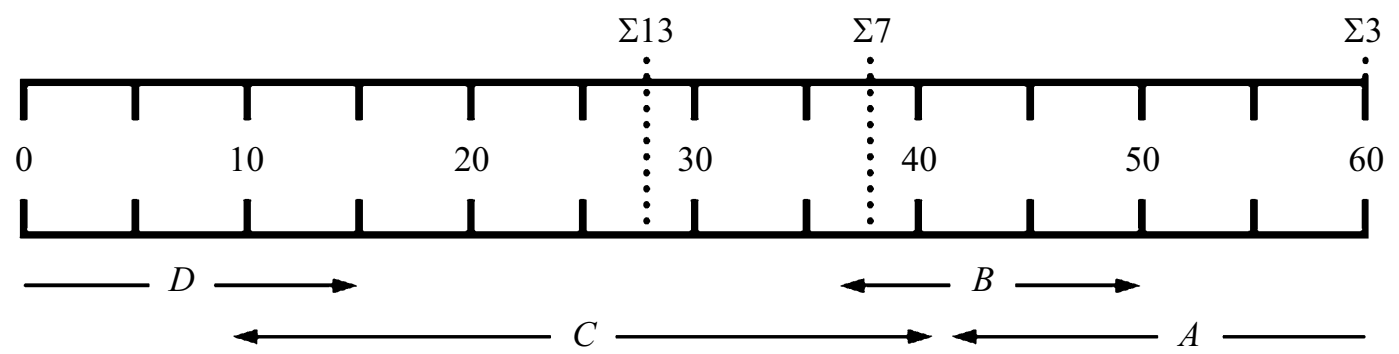

Рис. 12. „Шкала“ базовых структурных элементов ГЗ с осью разориентации [111].

то логично предположить, что и границы общего типа так же составлены из них. Анализ структуры ГЗ общего типа позволил определить границы углов разориентации, в которых встречаются базовые структурные и их модификации (рис. 12). Из рисунка видно, что любая граница может состоять из одного или двух базовых структурных элементов (включая их модификации). Малоугловые границы с углами разориентации менее $9^{\circ}$ состоят из элементов типа $D$, перемежающихся участками структурного несоответствия. В диапазоне углов соответствующих переходу от мало- к большеугловым ГЗ состоят из чередующихся элементов типа $C$ и $D$, причем с уменьшением угла разориентации элементов $C$ становится меньше, а элементов $D-$ больше. Элемент типа $B$ находится в самом узком диапазоне углов разориентации, т.е. является промежуточным между элементами типа $A$ и $C$.

\section{5. Заключение}

В работе в рамках структурно-вакансионной модели рассчитана атомная структура ГЗ наклона с использованием парного потенциала Морзе и многочастичного потенциала Клери-Розато. Зависимости энергии ГЗ от угла разориентации имеют подобный характер, и отличаются друг от друга только абсолютными значениями энергии.

Анализ атомной структуры ГЗ позволил выделить базовые структурные элементы, из которых построены симметричные ГЗ наклона специального и общего типа. Для каждой оси разориентации выделено по четыре базовых структурных элемента. Ряд структурных элементов имеют модификации, полученные в результате внесения в область ГЗ дополнительных атомов и вакансий. Исследованы закономерности присутствия базовых структурных элементов в зависимости от угла разориентации.

Для ГЗ с осью разориентации [110] специальные границы $\Sigma 3(111), \Sigma 3(112)$ и $\Sigma 11(113)$ состоят из базовых структурных элементов одного типа $-A, B$ и $C$ соответственно. Структура остальных изученных ГЗ может быть представлена чередованием базовых структурных элементов $A, B, C$ и $D$.
Для ГЗ с осью разориентации [111] специальные границы $\Sigma 3(112), \Sigma 7(123)$ и $\Sigma 13(134)$ имеют низкие значения энергии и состоят из базовых структурных элементов одного типа $A, B$ и $C$ соответственно. Большеугловые ГЗ специального типа с углами $\Theta>35^{\circ}$ состоят из комбинации базовых структурных элементов $A$ и $B$ в определенном соотношении, а с углами $\Theta<35^{\circ}$ - только из базовых структурных элементов $C$.

Структура границ общего типа может быть представлена чередованием базовых структурных элементов в зависимости от оси и угла разориентации. Определены диапазоны углов в пределах которых встречаются те или иные базовые структурные элементы.

\section{Конфликт интересов}

Авторы заявляют, что у них нет конфликта интересов.

\section{Список литературы}

[1] А.В. Векман, Б.Ф. Демьянов. ФММ 120, 53 (2019).

[2] G.H. Bishop, B. Chalmers. Scripta Met. 2, 133 (1968).

[3] A.P. Sutton, V. Vitek. Phil. Trans. R. Soc. Lond. A 309, 1 (1983).

[4] J.D. Rittner, D.N. Seidman. Phys. Rev. B 54, 6999 (1996).

[5] O.H. Duparc, J.-Ph. Couzinie, J. Thibault-Penisson, S. Lartigue-Korinek, B. Decamps, L. Priester. Acta Mater. 55, 1791 (2007).

[6] А.В. Векман, Б.Ф. Демьянов. ФПСМ 14, 480 (2017).

[7] G.J. Wang, A.P. Sutton, V. Vitek. Acta Met. 32, 1093 (1984).

[8] D. Wolf. J. Mater. Res. 5, 1708 (1990).

[9] D. Wolf. Acla Met. 37, 1983 (1989).

[10] D. Wolf. Phil. Mag. A 62, 447 (1990).

[11] D. Wolf. Acta Met. Mater. 38, 781 (1990).

[12] J.J. Bean, K.P. McKenna. Acta Mater. 110, 246 (2016).

[13] E.R. Homer, S.M. Foiles, E.A. Holm, D.L. Olmsted. Acta Mater. 61, 1048 (2013).

[14] P. Deymier, A. Taiwo, G. Kalonji. Acfa Met. 35, 2719 (1987).

[15] A. Suzuki, Y. Mishin. Interface Sci. 11, 131 (2003).

[16] А.В. Векман, Б.Ф. Демьянов. ФТТ 62, 1997 (2020).

[17] А.С. Драгунов, Б.Ф. Демьянов, А.В. Векман. Изв. вузов. Физика 53, 82 (2010).

[18] А.С. Драгунов, А.В. Векман, Б.Ф. Демьянов. Ползуновский альманах 4-1, 29 (2011).

[19] А.В. Векман. Автореф. канд. дис. 01.04.07. Барнаул. (2000). $23 \mathrm{c}$. 
[20] А.В. Векман, Б.Ф. Демьянов, А.С. Драгунов. ФММ 116, 621 (2015).

[21] P.M. Morse. Phys. Rev. 34, 57 (1929).

[22] F. Cleri, V. Rosato. Phys. Rev. B 48, 22 (1993).

[23] А.И. Царегородцев, Н.В. Горлов, Б.Ф. Демьянов, М.Д. Старостенков. ФММ 58, 336 (1984).

[24] А.В. Векман, А.С. Драгунов, Н.В. Адарич, Б.Ф. Демьянов. Ползуновский альманах 3, 49 (2008).

[25] А.В. Векман, А.С. Драгунов, Б.Ф. Демьянов, Н.В. Адарич. Изв. вузов. Физика. 55, 65 (2012).

[26] Дж. Хирт, И. Лоте. Теория дислокаций. Атомиздат, М. (1972). $600 \mathrm{c}$.

[27] M.A. Tschopp, D.L. Mcdowell. Phil. Mag. 87, 3871 (2007).

[28] А.В. Векман, Б.Ф. Демьянов, А.С. Драгунов. Изв. АлтГУ 1, 99, 11 (2018).

Редактор Т.Н. Василевская 